\section{AB0029 ANTI INFLAMMATORY EFFECT OF PDE5 INHIBITION: SCOPE FOR A NEW POTENTIAL INDICATION IN SSC ASSOCIATED MYOSITIS}

C. Corinaldesi ${ }^{1,2}$, G. Abignano ${ }^{1,3}$, C. Antinozzi ${ }^{2}$, V. Riccieri ${ }^{4}$, G. Valesini ${ }^{4}$ M. Vasile ${ }^{4}$, F. Marampon ${ }^{2}$, A. Lenzi ${ }^{4}$, K. Ballard ${ }^{5}$, F. Del Galdo ${ }^{1,3}$, C. Crescioli ${ }^{2}$. ${ }^{1}$ Leeds Institute of Rheumatic and Musculoskeletal Medicine, University of Leeds, Leeds, United Kingdom; ${ }^{2}$ University of Rome Foro Italico, Rome, Italy; ${ }^{3}$ NIHR Leeds Musculoskeletal Biomedical Research Unit, Leeds Teaching Hospitals NHS Trust, Leeds, United Kingdom; ${ }^{4}$ Sapienza University of Rome, Rome, Italy; ${ }^{5}$ Myriad RBM, Austin-TX, United States

Background: Skeletal muscle damage can occur as clinical manifestation of Systemic Sclerosis (SSc) [1], and it is known to be associated to type I IFN pathway activation [2]. The type I IFN-induced chemokine CXCL10 is associated with a more severe SSc prognosis and skeletal muscle disease [3,4], and it has been reported to play a role in inflammatory myopathy (IM) and in diabetic cardiomyopathy (DCM) $[5,6]$. In DCM the phosphodiesterase type 5 inhibitor (PDE5i) sildenafil significantly decreased CXCL10 systemic and cellular release [7]. In SSc, sildenafil is used to treat pulmonary artery hypertension (PAH) and digital ulcers disease.

Objectives: To determine the serum levels of CXCL10 in SSc patients with or without muscle involvement and treated or not with sildenafil. To determine the role of sildenafil on IFN $\gamma+$ TNF $\alpha$-induced CXCL10 release in human skeletal muscle cells (Hfsmc).

Methods: Sera from 109 patients fulfilling ACR/EULAR 2013 classification criteria for SSc and 34 age and gender matched healthy controls $(\mathrm{HC})$ were analysed by multiplexed, bead-based immunoassay. Hfsmc were cultured and analysed as previously described [5].

Results: CXCL10 serum level was significantly higher in SSc vs. HC $602.1 \pm 155.1$ $\mathrm{pg} / \mathrm{ml}$ vs $197.5 \pm 14.9 \mathrm{pg} / \mathrm{ml}, \mathrm{P}<0.001)$ independently of diffuse or limited clinical subset $(p>0.05)$; the presence of sildenafil in therapeutic regimen was associated with lower serum CXCL10 $(455.2 \pm 211.8 \mathrm{pg} / \mathrm{ml}$ vs $633.1 \pm 183.02 \mathrm{pg} / \mathrm{ml}, \mathrm{P}<0.05)$. CXCL10 was significantly higher in patients with increased Creatine Kinase (CK) $(2703 \pm 2172$ vs $454 \pm 82.51, \mathrm{P}<0.01)$ and its concentration strongly correlated with the levels of $\mathrm{CK}(\mathrm{r}=0.986, \mathrm{P}<0.001)$ and with Medsger muscle Severity score $(r=0.445, \mathrm{P}<0.001)$. In vitro, sildenafil suppressed IFN $\gamma+\mathrm{TNF} \alpha$-induced CXCL10 release by Hfsmc in a dose dependent manner, down to $50 \%$ secretion at $1 \mathrm{mM}(\mathrm{P}<0.05)$. The inhibition of $\mathrm{CXCL} 10$ secretion was associated with significant reduction in cytokine-induced STAT1, NFKB and JNK phosphorylation $(\mathrm{P}<0.01)$.

Conclusions: High CXCL10 level is associated with SSc independently from local or diffuse clinical subset and is lower in patients assuming sildenafil independently of other therapies. The strong correlation of CXCL10 and severity of muscle damage as assessed by serum CK and Medsger Muscle severity score, strongly indicate/confirm the involvement of IFN-I pathway activation during myositis in SSc. The direct inhibitory effect of PDE5 inhibitor Sildenafil on proinflammatory induced $\mathrm{CXCL} 10$ secretion warrant further research on the potential role of PDE5 inhibitors as disease modifying agents in SSC.

\section{References:}

[1] Varga J et al., J Clin Invest 2007, 117: 557-67.

[2] Higgs BW et al., Ann Rheum Dis 2011, 70:2029-36.

[3] Corrado A, Clin Ter 2014, 165:e436-41.

[4] Liu X et al., Arthritis Rheum 2013, 65:226-35.

[5] Crescioli $C$ et al. Eur J Cell Biol. 2012,91:139-49.

[6] Di Luigi L et al., PLoS One. 2013 Oct 30:8(10):e77745.

[7] Di Luigi et al., Inflammation 2016,39(3):1238-52.

Disclosure of Interest: None declared

DOI: 10.1136/annrheumdis-2017-eular.6233

\section{AB0030 THE EFFECT OF SILDENAFIL AND ILOPROST ON CXCL10 LEVEL IN SYSTEMIC SCLEROSIS: IN VIVO AND IN VITRO COMPARISON}

C. Antinozzi ${ }^{1}$, C. Corinaldesi ${ }^{2}$, F. Marampon ${ }^{1}$, V. Riccieri ${ }^{3}$, G. Valesini $^{3}{ }^{3}$ M. Vasile ${ }^{3}$, A. Lenzi ${ }^{4}$, F. Del Galdo ${ }^{5}$, C. Crescioli ${ }^{1}{ }^{1}$ University of Rome, Foro Italico, Rome, Italy; ${ }^{2}$ University of Leeds, Leeds, United Kingdom; ${ }^{3}$ University of Rome, Sapienza; ${ }^{4}$ University of Rome Sapienza, Rome, Italy; ${ }^{5}$ Univerisity of Leeds, Leeds, United Kingdom

Background: Th1 cell/cytokine repertoire contributes to systemic sclerosis (SSc) pathogenesis from early autoimmune/vascular stages while Th2 dominance prevails later, when (multi)organ fibrosis occurs [1]. The Th1-type chemokine IFN $\gamma$-induced $10 \mathrm{kDa}$ protein (CXCL10), involved in several autoimmune diseases (thyroiditis, rheumatoid arthritis, systemic lupus erythematosus, inflammatory myopathy) exerts detrimental effects at systemic and tissue/cell level; it is reported in association with more severe SSc prognosis [2-4]

Objectives: To compare circulating CXCL10 level in SSc patients starting iloprost (I), prostacyclin analogue with or without sildenafil (S), phosphodiesterase type 5 inhibitor, both vasoactive drugs used in SSc for Raynaud's phenomenon digital ischemic ulcers and/or secondary pulmonary arterial hypertension; SSc subjects under immunosuppressants (DMARDs) or corticosteroids (CCs) - first choice treatments at disease onset - before and after I were also evaluated. Intracellular path activation underlying Th1 cytokine-induced CXCL10 release by human skeletal muscle cells (Hfsmc), endothelial cells (Hfaec), cardiomyocytes (Hfcm) and fibroblasts (hFbs) were compared after iloprost or sildenafil.

Methods: Sera of 27 SSc patients satisfying ACR/EULAR 2013 classification criteria for SSc were analyzed by ELISA before (T0, baseline) and 3 months after I intake (T3) vs. 15 age/gender-matched healthy subjects. Protein extracts from different human cell types were tested by Western blot for IFN $\gamma+\mathrm{TNF} \alpha$-induced NFkB, Stat1, JNK activation after S or I.

Results: CXCL10 serum level was higher in all SSc under each drug/drug combination (range $\sim 300-500 \mathrm{pg} / \mathrm{ml}$ ) vs. healthy subjects $(\sim 150 \mathrm{pg} / \mathrm{ml}, \mathrm{P}<0.05)$. I intake did not modify baseline CXCL10 in SSc subjects taking only I, DMARDs or CCs, while it significantly decreased CXCL10 in subjects under $S(\sim 150 \mathrm{pg} / \mathrm{ml}$, $\mathrm{P}<0.05)$. In Hfsmc S-induced inhibition of Stat-1/NFkB/JNK phosphorylation was higher vs. I $(67 / 66 / 66 \% \mathrm{~S}$ vs. $28 / 30 / 38 \%$ I $\mathrm{P}<0.01)$. In Hfaec inhibition of Stat1/NFkB phosphorylation was higher and virtually prevented with I (99/92\% I vs. $58 / 66 \% \mathrm{~S} P<0.01$ ); conversely S-induced inhibition on JNK activation was significantly higher ( $66 \%$ S vs. $20 \%$ I P $<0.01)$. In Hfcm I-induced inhibition was stronger on Stat $1 / J N K$ phosphorylation $(67 / 83 \%$ I vs. $33 / 52 \% S \mathrm{P}<0.05 / \mathrm{P}<0.01)$, while inhibition on NFkB was similar $(70 \%$ I vs. $71 \% \mathrm{~S})$. In $\mathrm{hFbs}$, neither $\mathrm{S}$ nor I affected IFN $\gamma+\mathrm{TNF} \alpha$-induced activation of each analyzed path.

Conclusions: Our in vivo results show that $S$ and I combination is the more effective in targeting circulating CXCL10 in SSc patients. Our in vitro findings show a different inhibitory drug-induced effect onto paths underlying CXCL10 production, depending on cellular/intracellular targets. Thus, we suggest I and $S$ combination as potential pharmacological tool in SSc not limited to treat vascular dysfunction but likely helpful to control different cell/tissue involvement and damage.

\section{References:}

[1] Pattanaik D et al., Front Immunol 2015.

[2] Eloranta ML et al., Ann Rheum Dis 2010.

[3] Corrado A, Clin Ter 2014.

[4] Liu X et al., Arthritis Rheum 2013.

Disclosure of Interest: None declared

DOI: 10.1136/annrheumdis-2017-eular.6638

\section{AB0031 IL37 INHIBITS GAG RELEASE FROM HUMAN OA CARTILAGE EXPLANTS}

E. Van Geffen, A. van Caam, H. van Beuningen, E. Vitters, C. Dinarello, E. Blaney Davidson, P. van der Kraan. Radboud Univeristy Medical Center, Nijmegen, Netherlands

Background: In healthy cartilage, there is a balance between anabolic and catabolic activities of chondrocytes that maintains the functional integrity of the extracellular matrix. However, during osteoarthritis $(\mathrm{OA})$, chondrocytes become more catabolically active and express increased levels of matrix degrading enzymes, such as MMPs and ADAMTSs. Increased MMP and ADAMTS activity results in a net loss of the extracellular matrix and therefore leads to cartilage damage. Previously, we found that the anti-inflammatory cytokine Interleukin 37 (IL37) is able to counter-regulate the catabolic status of chondrocytes by reducing the IL1 $\beta$-driven expression of pro-inflammatory cytokines and catabolic enzymes. Objectives: The goal of this study was to investigate, in human OA cartilage explants, the effect of IL37 on sulfated glycosaminoglycans (GAG) content and synthesis of extracellular matrix molecules and cartilage degrading enzymes to investigate its therapeutic potential in OA.

Methods: Human cartilage was obtained from eighteen OA patients undergoing total knee or hip arthroplasty. Biopsy punches of $4 \mathrm{~mm}$ in diameter were made to equalize explant size. After culturing overnight, explants were incubated for $48 \mathrm{~h}$ with three doses $(1,10$ or $100 \mathrm{ng} / \mathrm{ml}$ ) of recombinant-human IL37 (rhlL37). In the supernatant of the explant cultures, GAG release was measured with the DMB assay and levels of the ARGS neoepitope, which is one of the products of aggrecan degradation by ADAMTS5, were detected using Western Blot. Furthermore, gene and protein expression of extracellular matrix molecules and cartilage degrading enzymes were measured. Nitric oxide (NO), an important effector molecule that may suppress cartilage matrix synthesis, levels were measured in the supernatant of the explants culture using Griess reagens.

Results: Adding rhIL37 (100 ng/ml) to OA cartilage explants caused a highly significant reduction in GAG release to the supernatant of, on average, $32 \%$ in eighteen donors (Figure 1). Gene expression of the matrix molecules aggrecan and collagen type II was not affected, indicating that this effect of rhIL37 was not due to a loss of aggrecan synthesis. Another mechanism to prevent GAG release in cartilage is via inhibition of NO synthesis, but NO levels in the supernatant were comparable between rhIL37 treated groups and the control group. In contrast, after addition of rhIL37, ARGS neoeptiope levels, which reflect ADAMTS5 activity, were dose-dependently down regulated in the supernatant. Furthermore, protein analysis of the explants showed that rhIL37 reduced ADAMTS5 levels. These data indicate that IL37 interferes with the amount active matrix degrading enzymes in the cartilage matrix. However, gene expression of ADAMTS5 was not affected by rhIL37, indicating that the effect of IL37 on ADAMTS5 is post translational.

Conclusions: Our data show that rhIL37 reduces GAG release by OA cartilage explants. The mechanism behind this protective effect of IL37 probably runs via a reduction in ADAMTS5 abundance in the cartilage matrix, which is the main aggrecanase involved in OA This effect of IL37 on ADAMTS5 is probably post translational. Our data indicate that IL37 can maintain cartilage matrix integrity 